\title{
Cerebral Blood Oxygenation Changes INDUCED BY VISUAL STIMULATION IN HUMANS
}

\author{
Rüdiger Wenzel, ${ }^{\dagger}$ Hellmuth Obrig, ${ }^{\dagger}$ Jan Ruben ${ }^{\dagger}$ Kersten Villringer, ${ }^{\dagger}$ Andreas Thiel, ${ }^{*}$ \\ Johannes Bernarding, ${ }^{*}$ Ulrich Dirnagl, ${ }^{\dagger}$ and Arno Villringer ${ }^{\dagger}$ \\ ${ }^{\dagger}$ Humboldt-Universität, Neurologische Klinik der Charité, Berlin, Germany; ${ }^{*}$ Universitätsklinikum \\ Benjamin Franklin, Radiologische Klinik, Berlin, Germany; *Institut für Medizinische \\ Statistik und Informationsverarbeitung, UKBF, Berlin, Germany \\ (Paper JBO/NIR-08 received May 6, 1996; revised manuscript received Sept. 3, 1996; accepted for publication Sept. 10, 1996)
}

\begin{abstract}
We examined local changes of cerebral oxygenation in response to visual stimuli by means of near infrared spectroscopy. A sharply outlined colored moving stimulus which is expected to evoke a broad activation of the striate and prestriate cortex was presented to sixteen healthy subjects. Six of these subjects were also exposed to a colored stationary and a gray stationary stimulus. In two subjects the colored moving stimulus was tested against the colored stationary with an optode position presumably over area V5/MT. As a control condition, subjects performed a simple finger opposition task. Since the calcarine fissure varies greatly with respect to bony landmarks, optodes were positioned individually according to 3-D reconstructed magnetic resonance imaging (MRI). Concentration changes in oxyhemoglobin ([oxy-Hb]) and deoxyhemoglobin ([deoxy-Hb]) were continuously monitored with a temporal resolution of $1 \mathrm{~s}$, using an NIRO 500 (Hamamatsu Photonics, KK, Japan). In response to the visual stimulus, the grand average across all sixteen subjects resulted in a significant increase in [oxy-Hb] of $0.33 \pm 0.09$ arbitrary units (mean \pm S.E.M.) mirrored by a significant decrease in [deoxy-Hb] of $-0.18 \pm 0.02$ arbitrary units, while the motor control condition elicited no significant changes in any parameters. When the near infrared spectroscopy probes were positioned over area V5/MT, the drop of [deoxy-Hb] associated with the moving stimulus was significantly more pronounced than with the stationary stimulus in both subjects examined. No significant differences between the visual stimuli were observed at the optode position close to the calcarine fissure. The oxygenation changes observed in this study are consistent with the pattern we have reported for motor activation. They are in line with physiological considerations and functional MRI studies relying on blood oxygenation level-dependent contrast. () 1996 Society of Photo-Optical Instrumentation Engineers.
\end{abstract}

Keywords near infrared spectroscopy; cerebral hemodynamics; visual stimulation.

\section{INTRODUCTION}

The visual system has been extensively studied in humans using imaging methods. Along with their use in elucidating the organization of the visual system, visual stimuli have frequently been employed to study physiological phenomena relating to neurovascular coupling ${ }^{1-3}$ and to evaluate new imaging techniques. ${ }^{4-6}$

A number of studies on the visual system have used near infrared spectroscopy (NIRS), which is now an established technique for noninvasive determination of changes in oxygenation and hemodynamics (for an overview, see Chance ${ }^{7}$ ) in the human brain. These studies, however, have yielded conflicting results. Although all studies detected an

Preliminary results of the research in this paper were presented at the Human Brain Mapping Conference in Boston in June $1996 .{ }^{49}$

Address all correspondence to Rüdiger Wenzel. E-mail: wenzel@rz.charite.hu-berlin.de increase in oxyhemoglobin ([oxy-Hb]) during visual stimulation, two studies ${ }^{8,9}$ reported an increase in deoxyhemoglobin ([deoxy-Hb]), while Meek et al. ${ }^{10}$ recently found a decrease of [deoxy- $\left.\mathrm{Hb}\right]$ in about half of the subjects and an increase in the remaining ones. Only one study reported an increase in $[0 x y-\mathrm{Hb}]$, which was accompanied by a decrease in [deoxy-Hb] in all six subjects examined. ${ }^{11}$ No statistical evaluation of these data is, however, presented in the latter study. A new and interesting approach was introduced by Gratton and co-workers, ${ }^{12}$ who used a frequency domain spectrometer to measure signal changes elicited by visual stimulation with a high temporal resolution. The signals detected in this study are suggested to be directly related to neuronal activity.

The lack of a [deoxy- $\mathrm{Hb}$ ] decrease in previous studies is a surprising result, since functional magnetic resonance imaging (fMRI) studies based on

1083-3668/96/\$6.00 (C) 1996 SPIE 
blood oxygenation level-dependent (BOLD) contrast, which is thought to reflect a decrease in [deoxy-Hb], ${ }^{13,14}$ were able to precisely localize distinct cerebral areas involved in processing visual information. In addition, a model of functional activation in NIRS using a motor paradigm, which was established in our group, revealed a significant decrease in [deoxy-Hb]. ${ }^{15}$ We minimized localization problems by localizing the optical probes individually according to previously acquired 3-D-MRI, to evaluate whether a visual stimulus evokes a qualitatively different response pattern.

\section{MATERIALS AND METHODS}

The technique of NIRS relies on the application of a modified Lambert-Beer law to measured variations in attenuation in order to determine the corresponding changes in the concentration of tissue chromophores. The method has been explained in detail elsewhere. ${ }^{16}$ Briefly, attenuation of light by tissue depends on scatter and absorption and in a highly scattering medium like brain tissue, optical attenuation can be expressed as follows:

$$
\text { Attenuation }=\alpha c d B+G,
$$

where $\alpha$ is the specific extinction coefficient of the absorbing compound measured in $\mu$ molar ${ }^{-1} \mathrm{~cm}^{-1}, c$ is the concentration of the absorbing compound measured in micromolar concentrations and $d$ is the interoptode spacing measured in centimeters. $B$, the differential path length factor (DPF), ${ }^{17}$ accounts for the increased optical path length due to scattering and the additive term $G$ for scattering losses. Under the assumption that $d, B$, and $G$ remain constant during the measurement period, concentration changes can be calculated:

$$
\Delta c=\frac{\Delta \text { Attenuation }}{\alpha d B} .
$$

If several chromophores with distinguishable absorption spectra are of interest, changes in concentration of a number of chromophores can simultaneously be computed from the changes in attenuation at a number of wavelengths using an algorithm incorporating the relevant extinction coefficients for each wavelength and chromophore. ${ }^{18}$

The DPF for the adult head has been estimated by time-of-flight methods ${ }^{19,20}$ and in the frequency domain by phase-shift measurements. ${ }^{21}$ Duncan and co-workers, $^{21}$ investigated one hundred subjects, and reported a value of 6.26 (S.D. 0.88) at $807 \mathrm{~nm}$ for the adult head without gender difference. Concentration changes in our paper are presented in arbitrary units, since we did not determine the DPF individually. The reported values correspond to absolute concentration changes from an arbitrary zero at the start of each measurement period in micromolar concentrations, assuming a DPF of 6.26 to facilitate the comparison with previous studies.
We used an NIRO 500 (Hamamatsu Photonics $\mathrm{KK}$, Japan) to continuously measure concentration changes of [oxy-Hb] and [deoxy- $\mathrm{Hb}]$ through the intact skull in reflection mode. ${ }^{22,23}$ Light emitted from four pulsed laser diodes (wavelengths of 775, 825,850 , and $904 \mathrm{~nm}$ ) was carried to an optode attached to the subject's head via an optical fiber. Light emerging from the tissue was returned to a photomultiplier tube via a second optode. Data were acquired with a temporal resolution of $1 \mathrm{~s}$ and changes in optical densities were converted to changes in chromophore concentration according to the algorithm implemented in the near infrared spectrometer. $^{24}$

Sixteen healthy, right-handed adults aged 19 to 46 years were examined; (nine women and seven men mean age 27 years). Each subject gave informed consent to participate in the study. The visual stimulus consisted of a multicolored dodecahedron moving at $6 \mathrm{~cm} \mathrm{~s}^{-1}$ which was displayed on a computer monitor placed $2 \mathrm{~m}$ from the subject at eye level. We thereby intended to evoke a broad activation not only of $V 1 / V 2$, but also of secondary visual areas. Thirty seconds of stimulus were alternated with the presentation of a blank dark screen for $30 \mathrm{~s}$. As a control condition, subjects performed $30 \mathrm{~s}$ of a simple sequential finger opposition task with the right dominant hand. Each of the sixteen subjects completed ten to twelve consecutive cycles. To test the hypothesis that hemodynamic changes vary with the quality of the visual stimulus, in six of the sixteen subjects two other visual stimuli were tested: (1) the multicolored dodecahedron, which remained stationary and (2) the same dodecahedron shaded in gray. The three visual stimuli were displayed in balanced order.

The calcarine sulcus varies strongly in relation to cranial landmarks. ${ }^{25}$ Therefore, the optodes were horizontally positioned over the right occipital region at the level of the calcarine sulcus according to 3-D-reconstructed high-resolution MRI. The lightemitting optode was placed $1 \mathrm{~cm}$ to the right of the midline to avoid the sagittal sinus and the lightcollecting optode 3 to $4 \mathrm{~cm}$ laterally to the first. In two of the subjects the NIRS probes were also placed $4 \mathrm{~cm}$ laterally to this standard position to compare the moving colored stimulus with the colored stationary stimulus. All measurements were performed while subjects were lying in a dark, quiet room. After dark adaptation, the first stimulus was presented as soon as stable baselines for [oxy- $\mathrm{Hb}$ ] and [deoxy- $\mathrm{Hb}$ ] were reached.

Data were related to an arbitrary zero calculated from the $6 \mathrm{~s}$ prior to stimulation onset and averaged over all respective cycles. A time frame of $6 \mathrm{~s}$ representing the maximum increase of [oxy- $\mathrm{Hb}$ ] (see bars in Figure 1) was defined according to the grand average, to evaluate the response in the single subjects. 


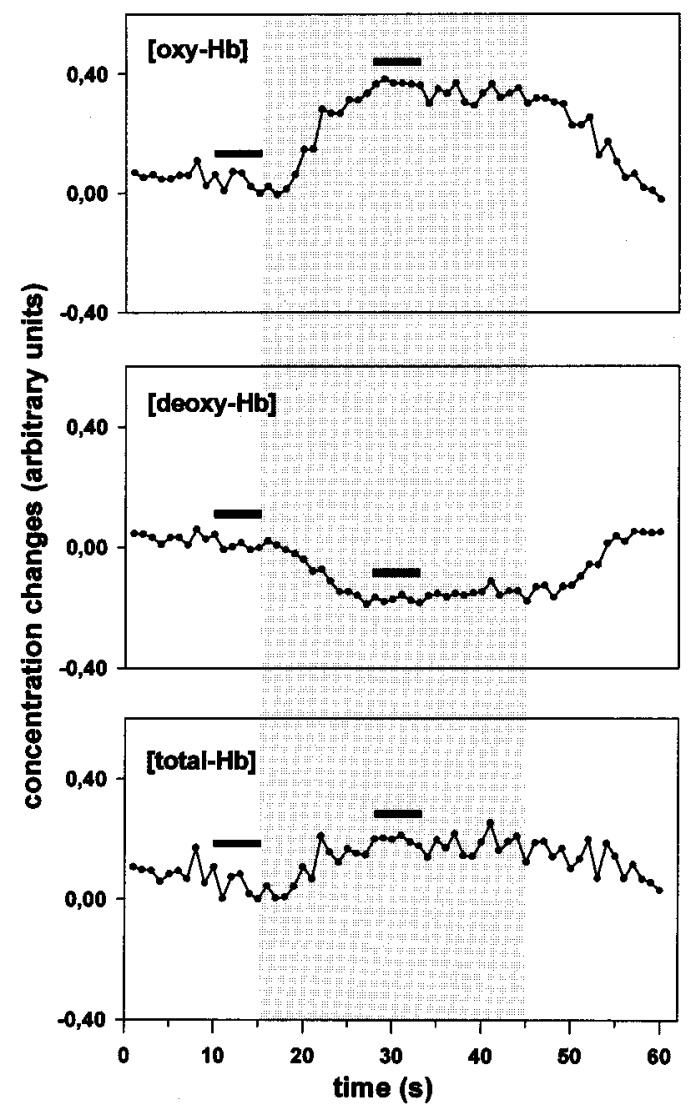

Fig. 1 Grand average over sixteen subjects. Gray-shaded area depicts the $30 \mathrm{~s}$ of visual stimulation. Changes in [oxy-Hb], [deoxy$\mathrm{Hb}$, and [total- $\mathrm{Hb}$ ] are given in arbitrary units, which correspond to micromolar concentration when assuming a DPF of 6.26. The horizontal bars denote the temporal windows that were averaged to characterize the response amplitude.

\section{RESULTS}

The grand average of all subjects is shown in Figure 1. $[\mathrm{Oxy}-\mathrm{Hb}]$ rises to its maximum during the first $14 \mathrm{~s}$ of stimulus presentation, followed by only a slight decrease during the stimulation period. Baseline values are reached $15 \mathrm{~s}$ after the end of the stimulation. This increase in [oxy-Hb] is mirrored by a decrease in [deoxy- $\mathrm{Hb}$ ] with an almost symmetrical time course. Since the increase in [oxy- $\mathrm{Hb}]$ exceeds the decrease in [deoxy-Hb], total hemoglobin $\left(\Delta[\right.$ total- $\mathrm{Hb}]=\Delta\left[\right.$ oxy-Hb] $\left.+\Delta[\text { deoxy-Hb }]^{26}\right)$ rises as well. When comparing the $6 \mathrm{~s}$ prior to stimulus onset with the $6 \mathrm{~s}$ period of maximal changes (also see bars in Figure 1), the mean ( \pm S.E.M.) change in [oxy- $\mathrm{Hb}$ ] was $0.33 \pm 0.09$ arbitrary units (a.u.), ${ }^{*}$ $-0.18 \pm 0.02$ a.u. in [deoxy-Hb] and $0.15 \pm 0.07$ a.u. in [total-Hb]. These changes were significant in all three parameters $(p=0.001$ for [oxy-Hb],$<0.001$ for [deoxy-Hb], and $<0.05$ for [total-Hb], paired $t$-test with $15 \mathrm{deg}$ of freedom). Mean changes during the

\footnotetext{
*Arbitrary units correspond to micromolar concentration assuming a DPF of 6.26 (Ref. 21) (see Materials and Methods).
}

last $5 \mathrm{~s}$ of the stimulation period were $0.33 \pm 0.08$ a.u. in [oxy-Hb] and $-0.15 \pm 0.03$ in [deoxy-Hb], which was not significantly different from the maximal changes. Hemoglobin concentrations in the motor control condition did not change significantly $(p=0.471$ for [oxy-Hb], 0.84 for [deoxy- $\mathrm{Hb}$ ], and 0.42 for [total-Hb]), but differed significantly from the values during visual stimulation, with $p=0.009$ for [oxy- $\mathrm{Hb}$ ] and $<0.001$ for [deoxy $-\mathrm{Hb}$ ].

Individual concentration changes are listed in Table 1. Using the same time frame as described above, mean differences for all cycles were calculated within each subject. Fourteen of the sixteen subjects showed an increase in [oxy-Hb] and fifteen a decrease in [deoxy-Hb]. $t$-Statistics revealed significance in nine and thirteen cases respectively. Three male subjects (subjects 3, 11, and 12 in Table 1) did not show significant concentration changes at all.

To evaluate whether the response of the three parameters examined is dependent on the quality of the visual stimulus, one-factorial ANOVAs for repeated measures with a polynomial contrast (SPSS) were performed. Neither the average of the six subjects, to whom the three different stimuli were presented, nor the individual means were revealed to be significantly different. However, in the two subjects in whom the second optode location was tested, the [deoxy-Hb] decrease was significantly more pronounced in response to the moving colored stimulus ( $p=0.003$ and 0.04 ).

\section{DISCUSSION}

In this study we were able to demonstrate an increase in [oxy-Hb] and a decrease in [deoxy-Hb] of about half the magnitude over the visual cortex during visual stimulation. This response pattern was not only seen in the grand average but also in thirteen of the sixteen single subjects. Three of the subjects did not show significant changes in response to the stimulus. Since an improper positioning of the optodes is unlikely, due to the MRIguided localization procedure, we think that signal losses by extracerebral tissue may account for this finding. In contrast to previous NIRS studies on the visual system, ${ }^{8-10}$ a significant decrease in [deoxy$\mathrm{Hb}$ ] in all subjects showing a stimulus-evoked response was observed.

The primary visual cortex or V1 does not typically extend onto the external aspect of the hemispheres, being mostly confined to the medial surface. ${ }^{27}$ Thus, the observed signal changes are likely to be derived from secondary visual areas such as V2, which lie in a horseshoe shape around $\mathrm{V} 1{ }^{27}$ A common feature shared by V1 and V2, which distinguishes them from other visual cortical areas, is that all submodalities of vision are represented in them. ${ }^{28}$ Other more specialized visual areas, which presumably have contributed to the signal changes, are the V3 complex and V4. V4, 
Table 1 Mean differences in [oxy-Hb], [deoxy-Hb] and [total- $\mathrm{Hb}$ ] ( \pm standard deviation-SD) between the $6 \mathrm{~s}$ prior to stimulation onset and 13 to $18 \mathrm{~s}$ after stimulation onset (see also bars in Fig. 1). The last three columns list the significance level $(* p<0.05, * * p<0.01, * * * p<0.001)$.

\begin{tabular}{|c|c|c|c|c|c|c|}
\hline Subject & $\Delta[\mathrm{oxy}-\mathrm{Hb}] \pm \mathrm{SD}$ & $\Delta[$ deoxy-Hb] $\pm \mathrm{SD}$ & $\Delta[$ total-Hb] $\pm \mathrm{SD}$ & $\Delta[\mathrm{oxy}-\mathrm{Hb}]$ & $\Delta[$ deoxy-Hb] & $\Delta[$ total- $\mathrm{Hb}]$ \\
\hline 1 & $0.09 \pm 0.71$ & $-0.11 \pm 0.10$ & $-0.02 \pm 0.77$ & - & $* * *$ & - \\
\hline 2 & $0.53 \pm 0.26$ & $-0.20 \pm 0.05$ & $0.33 \pm 0.23$ & $* * *$ & $* * *$ & $* * *$ \\
\hline 3 & $0.54 \pm 0.76$ & $-0.10 \pm 0.28$ & $0.45 \pm 0.99$ & - & - & - \\
\hline 4 & $1.16 \pm 0.21$ & $-0.33 \pm 0.09$ & $0.83 \pm 0.22$ & $* * *$ & $* * *$ & $* * *$ \\
\hline 5 & $0.37 \pm 0.27$ & $-0.29 \pm 0.17$ & $0.08 \pm 0.27$ & $* * *$ & $* * *$ & - \\
\hline 6 & $0.49 \pm 0.21$ & $-0.21 \pm 0.18$ & $0.28 \pm 0.22$ & $* * *$ & $* *$ & $* * *$ \\
\hline 7 & $0.11 \pm 0.43$ & $-0.08 \pm 0.09$ & $0.02 \pm 0.50$ & - & $* *$ & - \\
\hline 8 & $0.46 \pm 0.22$ & $-0.26 \pm 0.06$ & $0.19 \pm 0.21$ & $* * *$ & $* * *$ & $* *$ \\
\hline 9 & $0.34 \pm 0.58$ & $-0.21 \pm 0.09$ & $0.14 \pm 0.57$ & $*$ & $* * *$ & - \\
\hline 10 & $0.44 \pm 0.32$ & $-0.21 \pm 0.24$ & $0.23 \pm 0.37$ & $* * *$ & $* *$ & $* *$ \\
\hline 11 & $-0.29 \pm 0.70$ & $0.00 \pm 0.20$ & $-0.28 \pm 0.76$ & - & - & - \\
\hline 12 & $-0.20 \pm 0.41$ & $-0.05 \pm 0.21$ & $-0.25 \pm 0.57$ & - & - & - \\
\hline 13 & $0.17 \pm 0.35$ & $-0.12 \pm 0.14$ & $0.05 \pm 0.42$ & - & $*$ & - \\
\hline 14 & $0.48 \pm 0.28$ & $-0.24 \pm 0.11$ & $0.24 \pm 0.33$ & $* * *$ & $* * *$ & $*$ \\
\hline 15 & $0.09 \pm 0.72$ & $-0.15 \pm 0.16$ & $-0.07 \pm 0.83$ & - & $* *$ & - \\
\hline 16 & $0.49 \pm 0.21$ & $-0.31 \pm 0.05$ & $0.18 \pm 0.22$ & $* * *$ & $* * *$ & $*$ \\
\hline mean & 0.33 & -0.18 & 0.15 & & & \\
\hline S.E.M. & 0.085 & 0.024 & 0.067 & & & \\
\hline
\end{tabular}

located in the lingual and fusiform gyri of the prestriate cortex, has been demonstrated to be a color center, ${ }^{29}$ while V3 and V3A contain orientationselective cells. ${ }^{28}$ V5/MT, a motion-selective area, lies probably too anterior to have contributed to the changes at the standard optode position and was therefore tested in two subjects with a different localization of the NIRS probes.

The assumption that the pattern described reflects cerebral oxygenation changes evoked by a visual stimulus is supported by physiological considerations and by a comparison with results obtained by other functional imaging techniques (for a review, see Villringer and Dirnagl ${ }^{30}$ ). Fox and Raichle $^{2,31}$ reported regional uncoupling between cerebral blood flow and oxygen consumption during functional activation. Stimulus-induced focal augmentation of cerebral blood flow (50\%) by far exceeded the concomitant local increase in cerebral metabolic oxygen rate of about $5 \%$. The resulting focal "hyperoxygenation" corresponds to the increase in [oxy-Hb], accompanied by a decrease in [deoxy-Hb] as measured by NIRS.

In respect to the capillary bed, in vivo confocal laser-scanning microscopy ${ }^{32}$ and laser-Doppler flowmetry ${ }^{33}$ demonstrated increases in blood cell velocity and flux rate in response to hypercapnia and functional activation, respectively. This is consistent with a decrease in [deoxy-Hb], if the increase in blood cell velocity overcompensates for the increase in oxygen consumption. Finally, our finding of a decrease in [deoxy-Hb] is in line with functional MRI studies relying on BOLD contrast, since increases in signal intensities in gradient-echo MRI sensitive to susceptibility differences are ascribed to a decrease in deoxyhemoglobin. ${ }^{14}$ BOLD contrast was exploited in numerous studies to precisely localize distinct cerebral areas involved in visual processing., ${ }^{5,634-37}$ For a motor stimulus, a study simultaneously applying NIRS and BOLD contrast fMRI demonstrated that [deoxy- $\mathrm{Hb}$ ] changes as monitored by NIRS and signal intensity changes as measured by fMRI correlate well. ${ }^{38}$ In this context, although it is not significant, the slight positive slope of [deoxy- $\mathrm{Hb}$ ] after the maximum decreases despite sustained stimulation (see Figure 1) may be noteworthy since Frahm et al. ${ }^{39}$ recently reported a decreasing signal in cerebral blood oxygenation-sensitive MRI during prolonged visual stimulation. Further experiments with a variation 
of stimulus length are necessary to clarify this issue.

A model of functional activation using a motor paradigm revealed a pattern similar to that seen in this present study. ${ }^{15}$ There are, however, differences concerning the time course of the respective parameters. Changes in [oxy-Hb] exhibited a biphasic time course with a fast initial increase and a pronounced poststimulus undershoot in the motor study, which was not observed in the present study. The attempt to interpret these differences is subject to speculation. These are differences between both paradigms in the length of stimulation and resting periods $(30 \mathrm{~s} / 30 \mathrm{~s}$ versus $10 \mathrm{~s} / 50 \mathrm{~s}$ in the motor study), the quality of the stimulus, and the cortical region investigated. There is some evidence that the duration of rest can significantly influence hemodynamic responses. ${ }^{40} \mathrm{~A}$ visual stimulus is perceptive in nature, as opposed to an active motor task requiring a preparatory act. In addition, it should be borne in mind that different neuronal populations were investigated and it is still unclear whether neurovascular coupling is homogeneous within different cortical areas.

Our finding of a significant difference in the [deoxy- $\mathrm{Hb}$ ] decrease elicited by a moving colored and colored stationary stimulus in the two subjects studies with the optodes presumably located over area V5/MT underlines the specificity and the cortical origin of the detected signal changes, since it has been demonstrated by positron emission tomography (PET) and fMRI that area V5/MT responds much better to moving than to stationary visual stimuli. ${ }^{29,41,42}$ Nevertheless, the spatial resolution of the method is rather limited in the current approach and the exact estimation of the sample volume in NIRS is still an unsolved problem. Although one might have expected that prestriate areas contribute differently to the NIRS signal, depending on the quality of the visual stimulus, we failed to show differences between the three visual stimuli with the standard optode position.

To sum up, we demonstrated a consistent pattern of hemodynamic changes in the occipital cortex during visual stimulation. Differences in previous NIRS studies concerning [deoxy-Hb] changes may have been due to different optode positioning or the stimulus design. Bearing in mind the physiological associations, NIRS allows us to investigate oxygenation changes caused by functional activation in different cortical regions in humans. New approaches will overcome the current limitations and enable quantification by path length assessment on the basis of time or phase-resolved techniques $^{17,43}$ or water absorption spectra measurements. ${ }^{18,44}$ The use of a multiwavelength NIR spectrometer ${ }^{45}$ to measure the amplitude of a deoxy-Hb absorption peak in addition to the amplitude of water absorption features will permit an estimate of the absolute concentration of deoxy- $\mathrm{Hb}^{46}$ The limited spatial resolution of the method has been challenged by NIRS imaging as proposed by Benaron and Stevenson ${ }^{47}$ and Shinohara et al. ${ }^{48}$

\section{REFERENCES}

1. P. T. Fox and M. E. Raichle, "Stimulus rate determines regional brain blood flow in striate cortex," Ann. Neurol. 17, 303-305 (1985).

2. P. T. Fox, M. E. Raichle, M. A. Mintun, and C. Dence, "Nonoxidative glucose consumption during focal physiologic neural activity," Science 241, 462-464 (1988).

3. R. D. Frostig, E. E. Lieke, D. Y. Ts'o, and A. Grinvald, "Cortical functional architecture and local coupling between neuronal activity and the microcirculation revealed by in vivo high-resolution optical imaging of intrinsic signals," Proc. Natl. Acad. Sci. U.S.A. 87, 6082-6086 (1990).

4. J. W. Belliveau, D. N. Kennedy, Jr., R. C. McKinstry, B. R. Buchbinder, R. M. Weisskoff, M. S. Cohen, J. M. Vevea, T. J. Brady, and B. R. Rosen, "Functional mapping of the human visual cortex by magnetic resonance imaging," Science 254, 716-719 (1991).

5. K. K. Kwong, J. W. Belliveau, D. A. Chesler, I. E. Goldberg, R. M. Weisskoff, B. P. Poncelet, D. N. Kennedy, B. E. Hoppel, M. S. Cohen, R. Turner, H. M. Cheng, T. J. Brady, and B. R. Rosen, "Dynamic magnetic resonance imaging of human brain activity during primary sensory stimulation," Proc. Natl. Acad. Sci. U.S.A. 89, 5675-5679 (1992).

6. J. Frahm, H. Bruhn, K. D. Merboldt, and W. Hanicke, "Dynamic MR imaging of human brain oxygenation during rest and photic stimulation," J. Magn. Reson. Imaging 2, 501-505 (1992).

7. B. Chance, "Optical method," Ann. Rev. Biophys. Biophys. Chem. 20, 1-28 (1991).

8. T. Kato, A. Kamei, S. Takashima, and T. Ozaki, "Human visual cortical function during photic stimulation monitoring by means of near-infrared spectroscopy," J. Cereb. Blood Flow Metab. 13, 516-520 (1993).

9. Y. Hoshi and M. Tamura, "Dynamic multichannel nearinfrared optical imaging of human brain activity," J. Appl. Physiol. 75, 1842-1846 (1993).

10. J. H. Meek, C. E. Elwell, M. J. Khan, J. Romaya, J. S. Wyatt, D. T. Delpy, and S. Zeki, "Regional changes in cerebral haemodynamics as a result of a visual stimulus measured by near infrared spectroscopy," Proc. Roy. Soc. Lond. B 261, 351356 (1995).

11. A. Villringer, J. Planck, C. Hock, L. Schleinkofer, and U. Dirnagl, "Near infrared spectroscopy (NIRS): a new tool to study hemodynamic changes during activation of brain function in human adults," Neurosci. Lett. 154, 101-104 (1993).

12. G. Gratton, P. M. Corballis, E. Cho, M. Fabiani, and D. C. Hood, "Shades of gray matter: noninvasive optical images of human brain responses during visual stimulation," Psychophysiology 32, 505-509 (1995).

13. K. R. Thulborn, J. C. Waterton, P. Styles, and G. K. Radda, "Rapid changes of blood oxygenation and blood flow by high-field 1H n.m.r." Biochem. Soc. Trans. 9, 233-234 (1981).

14. S. Ogawa, T. M. Lee, A. R. Kay, and D. W. Tank, "Brain magnetic resonance imaging with contrast dependent on blood oxygenation," Proc. Natl. Acad. Sci. U.S.A. 87, 98689872 (1990).

15. H. Obrig, C. Hirth, J. Junge-Hülsing, C. Döge, T. Wolf, U. Dirnagl, and A. Villringer, "Cerebral oxygenation changes in response to motor stimulation," J. Appl. Physiol. (1996) (in press).

16. M. Cope and D. T. Delpy, "System for long-term measurement of cerebral blood and tissue oxygenation on newborn infants by near infra-red transillumination," Med. Biol. Eng. Comput. 26, 289-294 (1988).

17. D. T. Delpy, M. Cope, P. van der Zee, S. Arridge, S. Wray, and J. Wyatt, "Estimation of optical pathlength through tissue from direct time of flight measurement," Phys. Med. Biol. 33, 1433-1442 (1988).

18. S. Wray, M. Cope, D. T. Delpy, J. S. Wyatt, and E. O. Reynolds, "Characterization of the near infrared absorption spectra of cytochrome aa3 and haemoglobin for the nonin- 
vasive monitoring of cerebral oxygenation," Biochim. Biophys. Acta 933, 184-192 (1988).

19. M. Essenpreis, M. Cope, C. E. Elwell, S. R. Arridge, P. van der Zee, and D. T. Delpy, "Wavelength dependence of the differential pathlength factor and the log slope in timeresolved tissue spectroscopy," Adv. Exp. Med. Biol. 333, 9-20 (1993).

20. P. van der Zee, M. Cope, S. R. Arridge, M. Essenpreis, L. A. Potter, A. D. Edwards, J. S. Wyatt, D. C. McCormick, S. C. Roth, E. O. Reynolds, and D. T. Delpy, "Experimentally measured optical pathlengths for the adult head, calf and forearm and the head of the newborn infant as a function of inter optode spacing," Adv. Exp. Med. Biol. 316, 143-153 (1992).

21. A. Duncan, J. H. Meek, M. Clemence, C. E. Elwell, L. Tyszczuk, M. Cope, and D. T. Delpy, "Optical pathlength measurements on adult head, calf and forearm and the head of the newborn infant using phase resolved optical spectroscopy," Phys. Med. Biol. 40, 295-304 (1995).

22. M. Ferrari, E. Zanette, I. Giannini, G. Sideri, C. Fieschi, and A. Carpi, "Effects of carotid artery compression test on regional cerebral blood volume, hemoglobin oxygen saturation and cytochrome-C-oxidase redox level in cerebrovascular patients," Adv. Exp. Med. Biol. 200, 213-221 (1986).

23. C. E. Elwell, M. Cope, A. D. Edwards, J. S. Wyatt, E. O. Reynolds, and D. T. Delpy, "Measurement of cerebral blood flow in adult humans using near infrared spectroscopymethodology and possible errors," Adv. Exp. Med. Biol. 317, 235-245 (1992).

24. C. E. Elwell, "A practical users guide to near infrared spectroscopy," pp. 127-131, Hamamatsu Photonics KK (1995).

25. H. Steinmetz, G. Furst, and B. U. Meyer, "Craniocerebral topography within the international 10-20 system," Electroencephalogr. Clin. Neurophysiol. 72, 499-506 (1989).

26. J. S. Wyatt, M. Cope, D. T. Delpy, S. Wray, and E. O. Reynolds, "Quantification of cerebral oxygenation and haemodynamics in sick newborn infants by near infrared spectrophotometry," Lancet 2, 1063-1066 (1986).

27. S. Clarke and J. Miklossy, "Occipital cortex in man: organization of callosal connections, related myelo- and cytoarchitecture, and putative boundaries of functional visual areas," J. Comp. Neurol. 298, 188-214 (1990).

28. S. Zeki, "Parallelism and functional specialization in human visual cortex," Cold Spring Harbor Symp. Quant. Biol. 55, 651661 (1990).

29. S. Zeki, J. D. Watson, C. J. Lueck, K. J. Friston, C. Kennard, and R. S. Frackowiak, "A direct demonstration of functional specialization in human visual cortex," J. Neurosci. 11, 641649 (1991).

30. A. Villringer and U. Dirnagl, "Coupling of brain activity and cerebral blood flow: basis of functional neuroimaging," Cerebrovasc. Brain Metab. Rev. 7, 240-276 (1995).

31. P. T. Fox and M. E. Raichle, "Focal physiological uncoupling of cerebral blood flow and oxidative metabolism during somatosensory stimulation in human subjects," Proc. Natl. Acad. Sci. U.S.A. 83, 1140-1144 (1986).

32. A. Villringer, A. Them, U. Lindauer, K. Einhaupl, and U. Dirnagl, "Capillary perfusion of the rat brain cortex. An in vivo confocal microscopy study," Circ. Res. 75, 55-62 (1994).

33. U. Lindauer, A. Villringer, and U. Dirnagl, "Characterization of CBF response to somatosensory stimulation: model and influence of anesthetics," Am. J. Physiol. 264, H1223-8 (1993).

34. J. Frahm, K. D. Merboldt, and W. Hanicke, "Functional MRI of human brain activation at high spatial resolution," Magn. Reson. Med. 29, 139-144 (1993).
35. R. Turner, P. Jezzard, H. Wen, K. K. Kwong, D. Le Bihan, T. Zeffiro, and R. S. Balaban, "Functional mapping of the human visual cortex at 4 and 1.5 tesla using deoxygenation contrast EPI," Magn. Reson. Med. 29, 277-279 (1993).

36. A. Kleinschmidt, K. D. Merboldt, W. Hanicke, H. Steinmetz, and J. Frahm, "Correlational imaging of thalamocortical coupling in the primary visual pathway of the human brain," J. Cereb. Blood Flow Metab. 14, 952-957 (1994).

37. M. I. Sereno, A. M. Dale, J. B. Reppas, K. K. Kwong, J. W. Belliveau, T. J. Brady, B. R. Rosen, and R. B. Tootell, "Borders of multiple visual areas in humans revealed by functional magnetic resonance imaging,"' Science 268, 889-893 (1995).

38. A. Kleinschmidt, H. Obrig, M. Requardt, K. D. Merboldt, U. Dirnagl, A. Villringer, and J. Frahm, "Simultaneous recording of cerebral blood oxygenation changes during human brain activation by MRI and near-infrared spectroscopy," $J$. Cereb. Blood Flow Metab. 16, 817-826 (1996).

39. J. Frahm, G. Krüger, K. D. Merboldt, and A. Kleinschmidt, "Dynamic uncoupling and recoupling of perfusion and oxidative metabolism during focal brain activation in man," MRM 35, 143-148 (1996).

40. H. Obrig, T. Wolf, C. Döge, J. Junge-Hülsing, U. Dirnagl, and A. Villringer, "Length of resting period between stimulation cycles modulates hemodynamic response to a motor stimulus," Adv. Exp. Med. Biol. (1995) (in press).

41. J. D. Watson, R. Myers, R. S. Frackowiak, J. V. Hajnal, R. P. Woods, J. C. Mazziotta, S. Shipp, and S. Zeki, "Area V5 of the human brain: evidence from a combined study using positron emission tomography and magnetic resonance imaging," Cereb. Cortex 3, 79-94 (1993).

42. R. B. Tootell, J. B. Reppas, K. K. Kwong, R. Malach, R. T. Born, T. J. Brady, B. R. Rosen, and J. W. Belliveau, "Functional analysis of human MT and related visual cortical areas using magnetic resonance imaging," J. Neurosci. 15, 3215-3230 (1995).

43. B. Chance, M. Maris, J. Sorge, and M. Z. Zhang, "A phase modulation system for dual wavelength difference spectroscopy of hemoglobin deoxygenation in tissue," Proc. SPIE 1204, 481-491 (1990).

44. S. J. Matcher, M. Cope, and D. T. Delpy, "Use of the water absorption spectrum to quantify tissue chromophore concentration changes in near-infrared spectroscopy," Phys. Med. Biol. 39, 177-196 (1994).

45. M. Cope, D. T. Delpy, S. Wray, J. S. Wyatt, and E. O. Reynolds, "A CCD spectrophotometer to quantitate the concentration of chromophores in living tissue utilising the absorption peak of water at $975 \mathrm{~nm}, "$ Adv. Exp. Med. Biol. 248, 33-40 (1989).

46. S. J. Matcher and C. E. Cooper, "Absolute quantification of deoxyhaemoglobin concentration in tissue near infrared spectroscopy," Phys. Med. Biol. 39, 1295-1312 (1994).

47. D. A. Benaron and D. K. Stevenson, "Optical time-of-flight and absorbance imaging of biologic media," Science 259, 1463-1466 (1993).

48. Y. Shinohara, S. Takagi, N. Shinohara, F. Kawaguchi, Y. Itoh, Y. Yamashita, and A. Maki "Optical CT imaging of hemoglobin oxygen-saturation using dual-wavelength time gate technique," Adv. Exp. Med. Biol. 333, 43-46 (1993).

49. R. Wenzel, H. Obrig, J. Ruben, K. Villringer, C. Hirth, A. Thiel, and A. Villringer, "Haemodynamic changes induced by visual stimulation as measured with Near-Infrared Spectroscopy," Neuroimage 3, S302 (1996) (abstract). 